\title{
US delays testing anthrax vaccine for children
}

$\mathrm{F}$ ederal advisers have laid down a rigorous regimen that must be met before the United States government approves anthrax vaccine trials on children, all but ensuring no such tests will enlist youngsters any time soon.

It's but one sign that once-rampant fears of another catastrophic terrorist assault on US soil are receding, more than a decade after the attacks of Sept. 11, 2001.

The Presidential Commission for the Study of Bioethical Issues weighed in Mar. 19 on a question that vexes policy-makers as they consider how to protect children from a bioterrorism attack that might never happen, but could be devastating if it did.

In a report certain to carry clout with Health and Human Services Secretary, Kathleen Sebelius, who requested it, the commission said no anthrax vaccine trials involving children should take place until future studies confirm that the children would face no more than "minimal risk" - roughly equivalent to the danger from a routine medical checkup (http://bioethics.gov $/ \mathrm{cms} /$ sites/default/files/PCSBI_Pediatric -MCM_2.pdf).

Prevailing ethical standards call for children to be the subjects of medical trials only if those same youngsters stand to benefit. That's not the case with anthrax, a killer unlikely to emerge except in a particularly audacious act of mass violence.

Just a week after the 2001 terrorist attacks on the World Trade Center and Pentagon, anthrax spores sent through the mail killed 5 people, sickened 17 and scared practically everyone. The murders were blamed years later on a disturbed federal scientist, not on terrorists from abroad. Yet the episode showed the capacity to distribute lethal amounts of the bacteria widely and cheaply.

In one plausible doomsday scenario the US government has calculated, an anthrax attack theoretically exposed

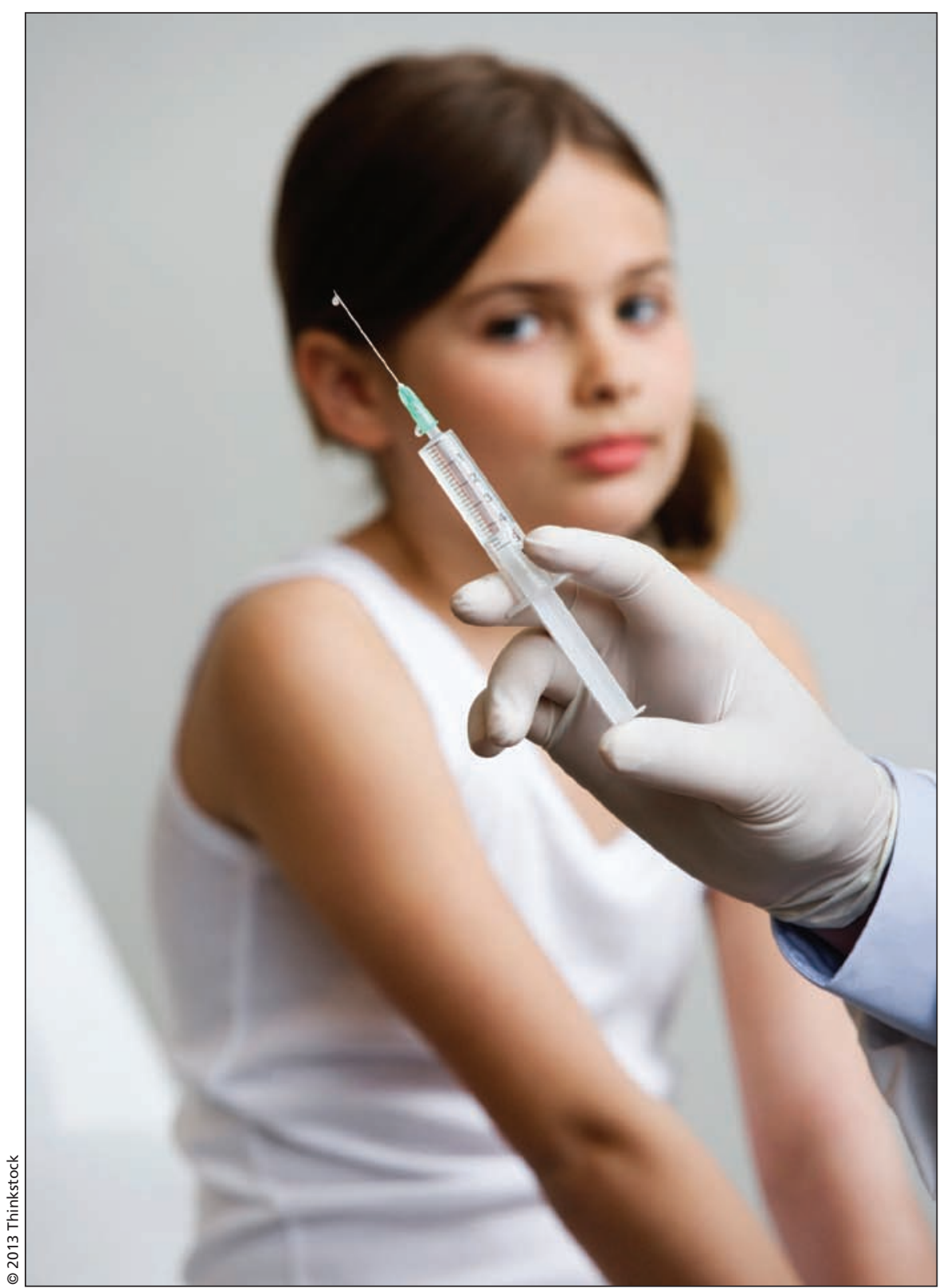

A US commission recommends that no anthrax vaccine trials involving children take place until future studies confirm that the children would face no more than "minimal risk" - roughly equivalent to the danger from a routine medical checkup.

nearly two million children and five million adults.

Proponents of trials argue the country cannot wait until an attack happens before figuring out whether a vaccine already approved for adults is also effective and safe for kids - and in what doses.
All of that prompted the commission to carve a careful path.

Commissioners recommended that researchers gather thorough information from tests on young adults before going any farther. After that, experimental vaccination carried out by the US government, or approved and funded by it, 
might be justified on the oldest children, then, in time, on progressively younger children.

As for where children might be found for trials should the studies go ahead in some form, the report suggests researchers turn to locations where participants are likely to be at an elevated risk of exposure to anthrax, such as urban areas near potential bioterrorism targets.

Researchers might also consider the children of emergency responders, the report says, because their parents are alert to the risks of terrorism and are more apt to want protection for their families that average citizens don't think about.

But the report cautions against selectively enrolling children in military families. Some soldiers already get the adult anthrax vaccine. But given the chain-of-command culture, military parents might take a request to include their children in a vaccine trial as an order. While service members have volunteered to be exposed to higher risks than most civilians, the report says, "their children have not."

The anthrax issue parallels a debate that unfolded in 2002 - when fears of a bioterrorism attack were acute - over a protocol to test smallpox vaccine in 40 children aged two to five. In that case, evidence of adverse reactions and secondary infections in adults suggested that a pediatric smallpox trial would subject children to more than minimal risk. The protocol was rejected, which means the vaccine Dryvax would not be available to children in an emergency.

Before children are enlisted for anthrax vaccine trials, the report says, "ethically sound modeling, testing with animals, and testing with the youngest adults must be completed to identify, understand, and characterize research risks."
Authorities are vigilant to the dangers of weaponized anthrax but, after years without a domestic terrorist attack, ordinary Americans aren't. "Respectable members of the public do not believe in the threat," Dr. John S. Parker, chairman of the National Biodefense Science Board, told the commission in earlier hearings.

People who are exposed to the spores are not without defences. In an attack using weaponized anthrax, potent antibiotics could deal with immediate exposure and a vaccine administered later could protect against pathogens lingering in a dormant state.

But what dosage children should get and what that would do to them and for them - are questions that may not be answered until that chaos comes, if it ever does. - Cal Woodward, Washington, DC

CMAJ 2013. DOI:10.1503/cmaj.109-4463 